\title{
Solvent cage spectroscopy
}

\author{
M. Kasha*, A. Sytnik and B. Dellinger** \\ Institute of Molecular Biophysics and Department of Chemistry, Florida State \\ University, Tallahassee, Florida 32306-3015
}

\begin{abstract}
Potential functions for photodissociation, excited state intramolecular torsion, and excited state twisting-intramolecular-charge-transfer are discussed in terms of sensitivity to solvent cage perturbation. A spectroscopic solvent cage theory based on a BornOppenheimer quantum mechanical framework is used for the justification of adducing viscous flow barriers as perturbations to the intrinsic molecular potentials. Experimental correlation of new spectroscopic phenomena is presented for each of the three potentials under perturbation.
\end{abstract}

\section{INTRODUCTION}

The principles of one-photon, single-molecule electronic excitation in terms of orbital configurations and spin assignments have been long established (ref. 1). In these systems unique $S_{1} \rightarrow S_{0}$ fluorescence and/or $T_{1} \rightarrow S_{0}$ low temperature phosphorescence have been observable. Following this came the era of multi-molecule excitation phenomena (molecular excitons, excimers, exciplexes, donor-acceptor complexes, as well as simultaneous transitions) and multiphoton excitation (biphotonic spectroscopy, infrared laser photochemistry). The latest period of molecular spectroscopic research has involved multi-channel excitation pathways involving gross molecular electronic and conformational changes. Recent decades have uncovered molecular systems which exhibit dual fluorescences (refs. 2,3) and even triple fluorescences (ref. 4) of various origins.

The interpretation of the dual fluorescence of 4-N, $\mathrm{N}^{\prime}$-dimethylaminobenzonitrile as a twisted-intramolecular-charge-transfer (TICT) phenomenon by Zbigniew Grabowski and co-workers (refs. 5,6 ) catalyzed the growth of interest in the subject. The TICT phenomenon has evolved into a major research development in contemporary spectroscopy (refs. 7,8). The large electronic structural and geometrical changes accompanying the multi-channel luminescence phenomena makes them ultra-sensitive to solvent cage effects. In this paper we shall survey several principal potential functions which arise and use those to exemplify the solvent cage control of the phenomena described.

\section{THE SPECTROSCOPIC SOLVENT CAGE MODEL}

The multi-channel excitation phenomena have brought to a focus numerous new researches in molecular dynamics, in which molecular friction plays a dominant rôle (refs. 9, 10,11). In addition, novel spectroscopic phenomena at the steady-state limits can be observed. These will be described here. The solvent cages which spectroscopists can probe extend greatly the range available to molecular dynamics studies which can be concerned mainly with the liquid solvent cages. In fact, the spectroscopic limits of interest are the liquid solvent cage at one extremum, and the rigidified solvent cage at the other. Highly contrasting phenomena may then be observed, such as fluorescence activation, or conversely, quenching. The rigid solvent cage can be a rigid glass matrix, a crystal (Shpol'ski isomorphic site), an adsorption surface, a zeolite or other inclusion complex such as a cyclo-dextrin or protein in-

\footnotetext{
*To whom queries should be addressed. Work done under Contract No. DE-FG05-87ER60517 of the Office of Health and Environmental Research, U. S. Department of Energy.

**University of Dayton Research Institute, University of Dayton, Dayton, Ohio 45469.
} 
clusion site. Fluorescence probes in such cages compared with their spectroscopic behavior in solution offer a wide range of perturbation effects of great theoretical interest and having wide practical applications.

In the spectroscopic solvent cage model for stationary state limits, we deal with potential surface perturbations. Such a concept, in which molecular micro-viscosity is adduced as a perturbation term to a molecular potential, requires a theoretical justification, since in dynamics terms it considers a time-dependent viscous flow as a static potential barrier addition to an equilibrium potential function. Dellinger and Kasha (refs. 12,13) have presented an analysis which uses a Born-Oppenheimer resolution of the molecular motions of solute and solvent. An outline of the assumptions and consequences of the Born-Oppenheimer spectroscopic theory of the solvent cage are epitomized in the following expressions. A further exposition of the quantum mechanical basis of this model has been presented recently (ref. 14).

$$
\begin{aligned}
& \mathrm{k}_{\mathrm{el}}>>\mathrm{k}_{\text {vib }}>\mathrm{k}_{\text {relax }}^{\prime}>\mathrm{k}_{\text {relax }}\left(\varepsilon_{\eta} \eta\right) \\
& \Psi_{\mathrm{abv}}\left(\mathrm{q}^{\prime}, \mathrm{q}, \mathrm{Q}_{\mathrm{R}}^{\prime}, \mathrm{Q}_{\mathrm{R}}\right)=\phi_{\mathrm{a}}\left(\mathrm{q}^{\prime}, \mathrm{q}, \mathrm{Q}_{\mathrm{R}}^{\prime}, \mathrm{Q}_{\mathrm{R}}\right) \cdot \theta_{\mathrm{ab}}\left(\mathrm{Q}_{\mathrm{R}}{ }^{\prime}, \mathrm{Q}_{\mathrm{R}}\right) \cdot \xi_{\mathrm{abv}}\left(\mathrm{Q}_{\mathrm{R}}\right) \\
& \mathrm{V}_{\text {molec-matrix }}(\mathrm{Q})=\mathrm{V}_{0 \text {,intrat }}(\mathrm{Q})+\mathrm{V}_{\text {inter }}(\mathrm{Q}) \\
& \mathrm{V}_{\text {inter }}(\mathrm{Q})=\varepsilon(\exp )\left[-\frac{1}{2} \mathrm{k}\left(\mathrm{Q}-\mathrm{Q}_{0}\right)^{2}\right] \\
& \frac{\partial \mathrm{V}}{\partial \mathrm{Q}}=0 \text { at } \mathrm{Q}_{0}
\end{aligned}
$$

Equation (1) expresses the Born-Oppenheimer starting premise of kinetic inequalities for rates of electron motion, molecular nuclear motion, solvent cage molecular nuclear motion (primed) and solute molecule nuclear motion (unprimed) at a critical coordinate. The last is a function of an energy barrier term and the corresponding molecular microviscosity.

Equation (2) adduces the separability of the total wavefunction as a product of independent wave functions for electronic, solvent cage molecule nuclear vibration, and solute molecule nuclear vibration.

Equation (3) adduces the perturbation theory consequence of the above, adding a solvent cage perturbation to the equilibrium potential.

Equation (4) is the expression for the Gaussian viscous flow barrier adopted, where $\varepsilon$ is the energy to surmount the barrier, and the exponential term gives the barrier shape.

Equation (5) is the special condition required to justify the kinetic inequality in the last two terms of Equation (1). Only when the slope of the unperturbed potential is horizontal can it be assumed that the rate of nuclear motion of solvent cage molecules and solute molecules are different.

\section{SOLVENT CAGE PERTURBATION OF THE DISSOCIATIVE POTENTIAL}

The spectroscopic solvent cage model has its simplest application to the Morse potential and the dissociative potential. An example will be presented here of the spectroscopic consequences of the solvent cage perturbation theory because of its illustrative nature, although the phenomenon to be described is not in the category of intramolecular multi-channel excitation modes. As Dellinger and Kasha (ref. 12) suggested, the effect on the Morse potential of solvent cage perturbations adduced by Equations (5) and (6) is a reduction in vibrational anharmonicity in the molecular vibrational mode represented by the Morse potential. This effect has been demonstrated for a special case by Mohammadi and Henry (ref. 15). 
For a dissociative potential case we choose the H-bonded complex of some diaza-aromatics, complexed to perfluoro-tertiary-butanol. These molecules offer the necessary special features: (a) the 9,10-diazaphenanthrene and 4-methylcinnoline have lowest $S_{0} \rightarrow S_{1}\left(n, \pi^{*}\right)$ absorption and fluorescence (a rarity) of easily observable quantum yield; (b) the alcohol chosen for hydrogen-bonding to the aza- nitrogen atom in each diaza-aromatic was chosen for greatest possible $\mathrm{H}$-bonding energy, offering a very stable H-bond. The principle of the experiment is illustrated by the schematic potentials of Fig. 1 . The ground

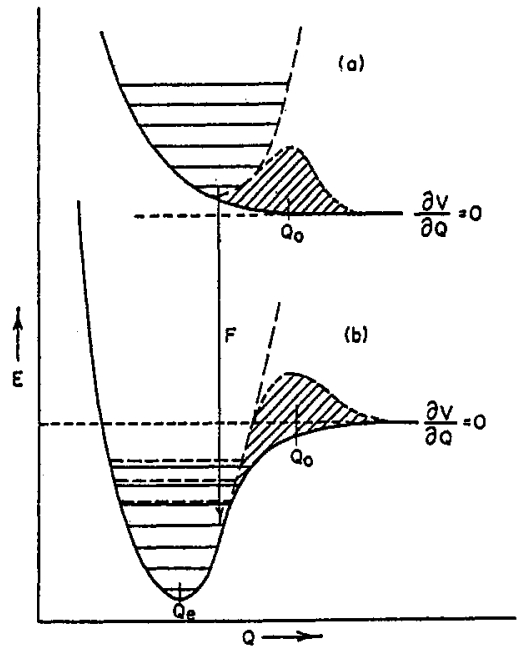

Fig. 1. Schematic potentials for H-bonding to an $\mathrm{N}$-atom lone-pair orbital in ground state $\mathrm{S}_{0}$ and first singlet excited state $S_{1}\left(n, \pi^{*}\right)$. state potential (solid curve) represents H-bond stabilization relative to the free molecule. The electronegativity of the $\mathrm{N}$-atom in the diazaaromatic results in an excess negative charge density on the $\mathrm{N}$-atom, whereas in the perfluorotertiary-butyl alcohol the $\mathrm{H}$-atom is strongly electropositive: a strong $\mathrm{H}$-bond results. Upon $\mathrm{n}$-orbital electron excitation, the $\mathrm{N}$-atom becomes $\mathrm{N}^{(+)}$, owing to the delocalization of the electronic charge upon $n \rightarrow \pi^{*}$ excitation. Consequently, strong repulsion in the first excited state of the complex is expected, and the potential function for the complex becomes repulsive (the covalent, and other, contributions to the H-bond likewise becoming negligible).

The experiment is carried out as follows. The spectra of 9,10-diazaphenanthrene and 4-methylcinnoline are determined in dry 3-methylpentane at $298 \mathrm{~K}$. Table I tabulates the results of the three-part experiment (full results to be published elsewhere, ref. 16).

TABLE 1. Absorption and Fluorescence Spectra $\left(S_{1} \leftrightarrow S_{0}\right),\left(n \rightarrow \pi^{*}\right)$ of diaza-aromatics and their H-bonded complex with perfluorotertiary butanol as a function of solvent cage perturbation in 3-methylpentane fluid solution (298K) and 3-methylpentane rigid glass matrix (77K).

\begin{tabular}{|c|c|c|c|c|c|c|c|}
\hline & \multirow[b]{2}{*}{$\mathrm{T}$} & \multicolumn{2}{|c|}{ Absorption } & \multirow[b]{2}{*}{ Spectrum } & \multicolumn{2}{|c|}{ Fluorescence } & \multirow[b]{2}{*}{ Spectrum } \\
\hline & & $\lambda_{\max }$ & $\lambda_{\text {oused }}$ & & $\lambda_{\text {oesect }}$ & $\lambda_{\max }$ & \\
\hline $\begin{array}{l}\text { (a) 9,10-Diazaphenanthrene } \\
\text { (b) H-bonded complex } \\
\text { (c) H-bonded complex }\end{array}$ & $\begin{array}{r}298 \mathrm{~K} \\
298 \mathrm{~K} \\
77 \mathrm{~K}\end{array}$ & $390 \mathrm{~nm}$ & $\begin{array}{l}450 \mathrm{~nm} \\
420\end{array}$ & $\begin{array}{l}\text { structured } \\
\text { diffuse }\end{array}$ & $\begin{array}{l}440 \mathrm{~nm} \\
440 \\
400\end{array}$ & $\begin{array}{l}480 \mathrm{~nm} \\
480 \\
440\end{array}$ & $\begin{array}{l}\text { structured } \\
\text { structured } \\
\text { diffuse }\end{array}$ \\
\hline $\begin{array}{l}\text { (d) 4-Methylcinnoline } \\
\text { (e) H-bonded complex } \\
\text { (f) H-bonded complex }\end{array}$ & $\begin{array}{r}298 \mathrm{~K} \\
298 \mathrm{~K} \\
77 \mathrm{~K}\end{array}$ & 385 & $\begin{array}{l}440 \\
400\end{array}$ & $\begin{array}{l}\text { structured } \\
\text { diffuse }\end{array}$ & $\begin{array}{l}430 \\
430 \\
375\end{array}$ & $\begin{array}{l}475 \\
475 \\
425\end{array}$ & $\begin{array}{l}\text { structured } \\
\text { structured } \\
\text { diffuse }\end{array}$ \\
\hline
\end{tabular}

(a,d) show sharply defined vibrational structure for the pure diaza-aromatic in dilute solution at $298 \mathrm{~K}$.

(b,e) show that typically blue-shifted $\mathrm{S}_{0} \rightarrow \mathrm{S}_{1}\left(\mathrm{n}, \pi^{*}\right)$ absorption (now diffuse) is observed, but for the H-bonded complexes (Structures I, II) the structured fluorescence emission of the uncomplexed molecule is observed. Thus, in Fig. 1 the dissociative potential is fully operative in the excited state in liquid 3-methylpentane solution at $298 \mathrm{~K}$ for $n \rightarrow \pi^{*}$ excitation. The fluorescing molecule is no longer complexed.

$(c, f)$ show that at $77 \mathrm{~K}$ when the H-bonded complex is excited in 3-methylpentane rigid glass matrix, the photodissociation is now blocked, and a diffuse blue-shifted fluorescence $\left[S_{1}\left(n, \pi^{*}\right) \rightarrow S_{0}\right]_{H-b o n d e d}$ for the complex is now observed. Thus, in the excited state in the rigid glass matrix the viscous-flow perturbation barrier becomes impenetrable, and fluorescence emission now occurs for the cage-stablized complex. 
An analogous result for a covalently-bonded photodissociation (perfluoroalkyl iodide) was reported by Brus and Bondybey (ref. 17), who demonstrated the conversion of a gas phase photodissociation to a structured fluorescence in rare gas and $\mathrm{N}_{2}$ solid matrix media, using an ad hoc partial cage potential.

THE TORSIONAL POTENTIAL

The torsional potential for internal twisting in molecules is well-known from the work of Mulliken and Roothaan (ref. 18) for the case of ethylene $\mathrm{C}=\mathrm{C}$ bond torsion. The idea and theory carry over to the stilbene molecule (1,2-diphenylethylene), except that an upper-state $\left(S_{2}\right)$ perturbation introduces a small intrinsic barrier near the $S_{1}$ excited state maximum of the torsional potential calculated by Orlandi and Siebrand (ref. 19). Figure 2 represents schematically the potential curves for $t$-stilbene in its first

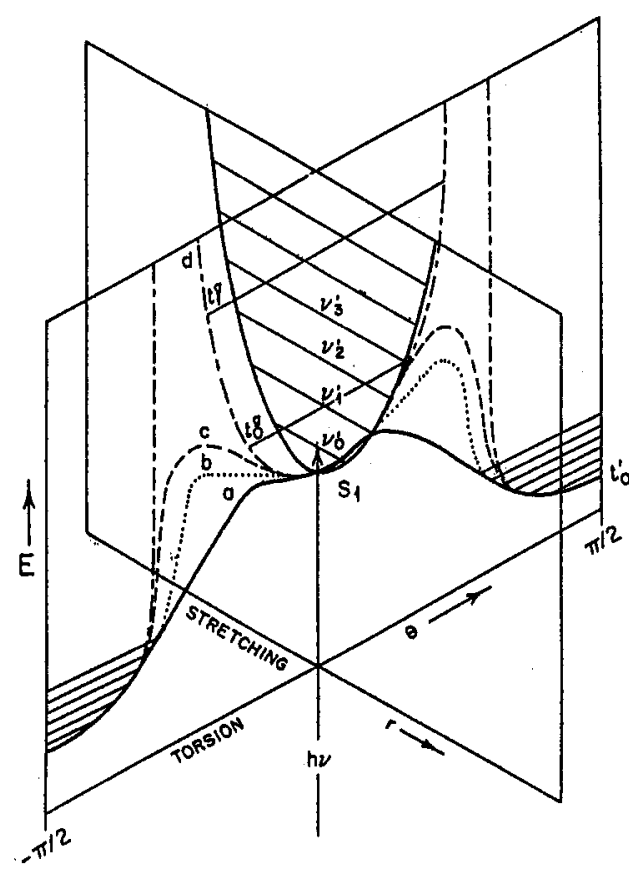

Fig. 2. Schematic potential curves for a harmonic stretching mode and a torsional mode in the $S_{1}$ excited state of a polyatomic molecule ( $t$-stilbene). $\pi \rightarrow \pi^{*}$ excited state $S_{1}$. Cross-sections of the potential hypersurface are chosen for a $C=C$ stretching vibration, and the $\mathrm{C}=\mathrm{C}$ torsional vibration. The stretching potential is depicted as a harmonic parabolic potential, as even a Morse or real potential would show little solvent cage perturbation near the zero-point level. The torsional potential has, except for the small intrinsic barrier $a$, essentially a quasi-stable minimum at $\theta$ $=0^{\circ}$. Thus, most molecules will very rapidly depopulate the "saddle-point" at $\theta=0^{\circ}$, and much attention has been paid to molecular dynamics studies of this rapid conformational change (Cf. ref. 9). A very small quantum yield of fluorescence from the zero-point levels of state $S_{1}$ is observable. This is crucial to our present report.

It was recognized long ago by Beale and Roe (ref. 20 ) that the first $U V$ absorption band of $t$-stilbene is dramatically altered upon comparing a liquid solution spectrum at $298 \mathrm{~K}$ with the $77 \mathrm{~K}$ rigid solvent matrix spectrum. One of the present authors adduced the solvent cage potential perturbation argument presented there, supported later by the Born-Oppenheimer basis (refs. 12,13). We shall develop this argument in a new direction here. As Fig. 2 adduces, the torsional potential is subject to solvent cage perturbations because the conditions specified by Equations 5,3 , and 4 exist. Thus, in Fig. 2, we depict the small intrinsic barrier of Orlandi and Siebrand (defining $\mathrm{Q}_{0}$ of Equations. 4 and 5) to which we add progressive solvent cage perturbation barriers $b$, and $c, \ldots .$. ; until in the rigid matrix we reach the infinite barrier $d$ against free torsion. Now the torsional mode becomes a very high-frequency, virtually inaccessible mode. The spectra we investigated offer us a chance to test the solvent-cage perturbation idea. The first series involves the $S_{0} \rightarrow S_{1}\left(\pi, \pi^{*}\right)$ absorption spectra of $t$-stilbene at $298 \mathrm{~K}$ in a series of solvents of increasing viscosity. According to Fig. 2 , the fluorescence emission should originate from the zero-point level of the $S_{1}$ state, and the Franck-Condon integrations involve the corresponding eigenfunction integrated over the products of the $v_{n}{ }^{\prime \prime}$ vibrational eigenfunctions for the $S_{0}$ state. Since the ground state has deep minima, the fluorescence spectral contours should be invariant with solvent viscosity. We find this to be the case, from $t$-stilbene in isopentane at $298 \mathrm{~K}$, to mineral oils, to glycerine, to methylmethacrylate rigid polymer films. The Franck-Condon envelope reveals a closely parallel intensity structure for the four observable vibronic bands of the fluorescence, and the band half-width (FWHM in current jargon) is relatively constant. Thus, virtually no observable solvent-cage perturbation on the fluorescence band is observed.

The absorption spectra in contrast prove to be very sensitive to solvent cage perturbations. The reasons can be clearly anticipated from a study of Fig. 2 . Obviously, the absorption to the stretching potential normal modes will be fairly immune to solvent cage perturbations. The Franck-Condon integration 
over the torsional mode eigenfunctions will sensitively reflect which (anti-)torsional barrier is in effect, $a, b, c, \ldots$ or $d$. Each one will present its own set of torsional eigenfunctions for the Franck-Condon integration. The Franck-Condon integration for absorptions from the zero-point levels of the $S_{0}$ state could then be over the stretching-vibrational eigenfunctions of $S_{1}$, the torsional eigenfunctions, and the corresponding cross-terms (occurring even in the absence of anharmonicity (ref. 21).

As expected, we find the first absorption band of $t$-stilbene to be extremely solvent-sensitive, with changes in general over-all band-shape, changes in Franck-Condon intensity of progressive $0^{\prime \prime}-0^{\prime}, 0^{\prime \prime}-1^{\prime}, 0^{\prime \prime}-2^{\prime}$ apparent vibronic bands. Especially prominent is the contraction in band width in the most viscous solvent in the series, isopentane, glycerine, methylmethacrylate solid polymer, all at $298 \mathrm{~K}$. The results are the more striking, when it is seen that the corresponding fluorescence band for the same conditions is invariant, with the same changes. For example, the absorption half-width (FWHM) of the solvent series are $6300,6000,4300 \mathrm{~cm}^{-1}$; whereas the corresponding fluorescence half-widths are essentially constant $\left(3900,3800,3700 \mathrm{~cm}^{-1}\right)$. Parallel to the Franck-Condon anomaly observed for the absorption spectroscopy is a dramatic fluorescence activation with increasing viscosity: from an almost negligible quantum yield of fluorescence in fluid solutions, the quantum yield approaches unity in the rigid polymer film, a rigid glass matrix, and in the molecular crystal (ref. 22). Additional studies on $77 \mathrm{~K}$ rigid glass spectroscopy also will be published (ref. 23).

\section{THE TWISTING-INTRAMOLECULAR-CHARGE-TRANSFER POTENTIAL}

The TICT phenomenon as described by Grabowski et al (ref. 6) takes as (a) a conformation of coplanar moieties for $S_{1}\left(\pi, \pi^{*}\right) \rightarrow S_{0}$ fluorescence and (b) as the origin of the $S_{1}{ }^{\prime}(C T) \rightarrow S_{0}$ second fluorescence a perpendicular conformation of charge transfer moieties (Fig. 3) We have proposed (ref. 4) as a quan-

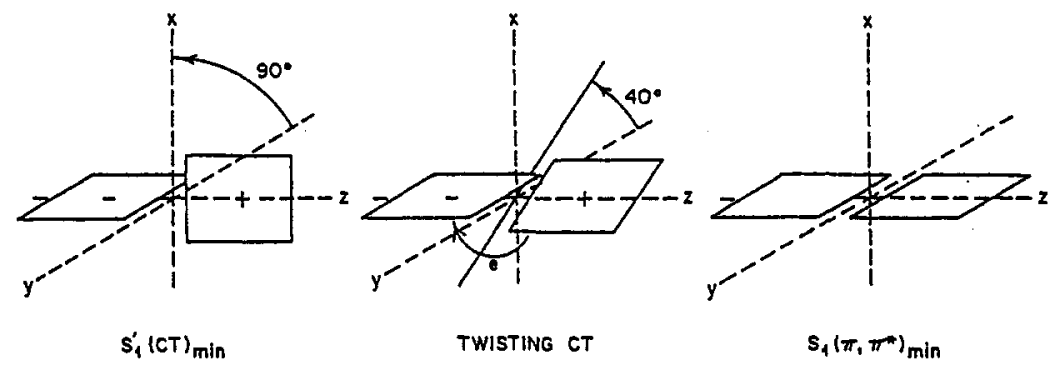

Fig. 3. Configurations of twisting moieties in TICT excitation.

tum mechanical starting point the $\mathrm{N}, \mathrm{N}^{\prime}$-dimethylamino-group twisting potentials calculated by Godfrey and Murrell (ref. 24). Our discussion (ref. 4) introduces a non-adiabatic region where the torsionally descending $S_{2}(C T)$ intersects with the rising $S_{1}\left(\pi, \pi^{*}\right)$ potential, as shown in Fig. 4. According to Murrell et al (ref. 24), $\sim 40^{\circ}$ is the torsional angle for their case of phenylamino-group twisting intersection $S_{1}\left(\pi, \pi^{*}\right)-S_{2}(C T)$. Thus, after $S_{0} \rightarrow$ $S_{1}\left(\pi, \pi^{*}\right)$ excitation, the molecule goes adiabatically along the potential and becomes at $\sim 40^{\circ}$ an $S_{1}{ }^{\prime}(\mathrm{CT})$ dipolar charge-transfer species (Fig. 3), a sudden polarization in the language of Salem (ref. 25). Thereupon, it relaxes to the torsional potential minimum. The new fluorescence $S_{1}{ }^{\prime}(C T) \rightarrow$ $\mathrm{S}_{0}(\mathrm{FC})$ is to higher vibronic states of the ground state, and thus there can generally be no converse absorption band. The dual fluorescence of tor-

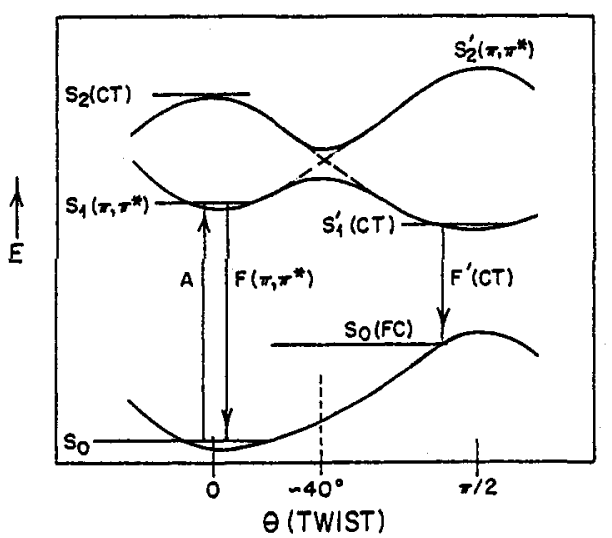

Fig. 4. Schematic twisting-intramolecular-charge-transfer potentials for sudden polarization from $S_{1} \rightarrow S_{1}{ }^{\prime}(C T)$. 
sionally capable molecules with donor-acceptor moieties, can thus be observed if the dielectric environment is favorable (usually a polar rather than a hydrocarbon solvent).

The solvent cage perturbation effects described in the previous section for the torsional potential (Fig. 2) can be expected to apply to the twisting-intra-molecular-charge-transfer (TICT) potential as well. The general principle is that a large amplitude intramolecular motion must be involved in order for the criteria of Equations 3, 4, and 5 to be effective. A molecule which can exemplify the sensitivity of TICT potential to solvent cage perturbation is the case of $\mathrm{N}$-methylbenzanilide. The $\mathrm{N}$-methylbenzanilide exhibits a strong TICT fluorescence, $S_{1}{ }^{\prime}(C T) \rightarrow S_{0}(F C)$ with $\lambda_{\max }$ at $510 \mathrm{~nm}$ in hydrocarbon solution at $298 \mathrm{~K}$ (ref. 26). At $77 \mathrm{~K}$ in a hydrocarbon fluorescence is quenched, as the rigid glass cage blocks the large amplitude motion required to reach the sudden polarization $S_{1}{ }^{\prime}(C T)$ curve. So only UV fluorescence for the $S_{1}\left(\pi, \pi^{*}\right)$ state at $\theta=0^{\circ}$ is seen. This is in contrast to the fluorescence activation observed for the case of $t$-stilbene, in which the rigid glass solvent cage restricts twisting, thus increasing the efficiency of $S_{1}\left(\pi, \pi^{*}\right) \rightarrow S_{0}$ fluorescence. Of course, in the TTCT case, the twisted configuration at $\theta=90^{\circ}$ must be reached for the $S_{1}{ }^{\prime}(C T) \rightarrow S_{0}(F C)$ second fluorescence $\left(F_{2}{ }^{\prime}\right)$ to be observed. Thus, a solvent cage restriction on internal torsion in this case quenches the TICT-state fluorescence. This can be used as a diagnostic tool to separate the second fluorescence $\left(\mathrm{F}_{2}\right)$ observed for the proton-transfer potential case, which is not affected by solvent cage restriction and is readily observed in rigid glass solutions at $77 \mathrm{~K}$ (ref. 27). These distinctions become especially important in unravelling the multi-channel mechanism cases, especially when triple fluorescence can be observed (ref. 4).

\section{REFERENCES}

1. Cf. M. Kasha, Radiation Res., Supplement 2, 243-275 (1960).

2. A. Weller, Z. Elektrochem., 60, 1144-1147 (1956).

3. E. Lippert, W. Lüder and H. Boos, Advan. Mol. Spectros., Vol. 1 (A. Mangini, Ed.), Pergamon Press, New York, pp. 443-457 (1962).

4. J. Heldt, D. Gormin and M. Kasha, Chem. Physics, 136, $321-334$ (1989).

5. K. Rotkiewicz, K. H. Grellmann and Z. R. Grabowski, Chem. Phys. Lett., 19, 315-318 (1973).

6. Z. R. Grabowski, K. Rotkiewicz, A. Siemiarczuk, D. J. Cowley and W. Baumann, Nouv. J. Chim. 3, 443-454 (1979).

7. W. Rettig, Angew. Chem. Int. Ed. English, 25, 971-988 (1986).

8. E. Lippert, W. Rettig, V. Bonacic-Koutecky, F. Heisel and J. A. Miehé, in Advances in Chemical Physics, 68 (I. Prigogine and S. A. Rice, Eds.), Wiley-Interscience, New York, pp. 1-173 (1987).

9. S. Abrash, S. Repinec and R. M. Hochstrasser, J. Chem. Phys., 93, 1041-1053 (1990).

10. B. Bagchi and G. R. Fleming, J. Phys. Chem., 94, 9-20 (1990).

11. A. Burshtein and D. Kivelson, Eds., Chemical Physics, 152, 1-222 (1991).

12. B. Dellinger and M. Kasha, Chem. Phys. Lett., 36, 410-414 (1975).

13. B. Dellinger and M. Kasha, Chem. Phys. Lett., 38, 9-14 (1976).

14. M. Kasha, D. Parthenopoulos and B. Dellinger, Int. J. Quantum Chem. (in Press, 1992).

15. M. A. Mohammadi and B. R. Henry, Proc. Nat. Acad. Sci. USA, 78, 686-688 (1981).

16. B. Dellinger and M. Kasha, This Laboratory (manuscript in preparation, 1992).

17. L. E. Brus and V. E. Bondybey, Chem. Phys. Lett., 36, 252-255 (1975).

18. R. S. Mulliken and C. C. J. Roothaan, Chem. Rev., 41, 219-231 (1947).

19. G. Orlandi and W. Siebrand, Chem. Phys, Lett., 30, 352-354 (1975).

20. R. N. Beale and E. M. F. Roe, J. Chem. Soc., 2755-2763 (1953).

21. C. A. Coulson, J. Duchesne and C. Manneback, in Contribution à l'Étude de la Structure Moléculaire (V. Henri Commemorative Volume) Desoer, Liége, pp. 33-46 (1948).

22. A. Sytnik and M. Kasha, This Laboratory (manuscript in preparation, 1992).

23. G.-Q. Tang and M. Kasha, This Laboratory (manuscript in preparation, 1992).

24. M. Godfrey and J. N. Murrell, Proc. Roy. Soc. London, A278, 71-90 (Cf. Fig. 8) (1969).

25. L. Salem, Accounts Chem. Res., 12, 87-92 (1979).

26. J. Heldt, D. Gormin and M. Kasha, J. Am. Chem. Soc., 110, 8255-8256 (1988).

27. D. McMorrow and M. Kasha, J. Phys. Chem., 88, 2235-2243 (1984). 